\title{
THE GELFAND SPECTRUM OF A NONCOMMUTATIVE C*-ALGEBRA: A TOPOS-THEORETIC APPROACH
}

\author{
CHRIS HEUNEN ${ }^{\otimes}$, NICOLAAS P. LANDSMAN, BAS SPITTERS and \\ SANDER WOLTERS
}

(Received 23 July 2010; accepted 21 December 2010)

Communicated by V. Mathai

Dedicated to Alan Carey, on the occasion of his 60th birthday

\begin{abstract}
We compare two influential ways of defining a generalized notion of space. The first, inspired by Gelfand duality, states that the category of 'noncommutative spaces' is the opposite of the category of $\mathrm{C}^{*}$-algebras. The second, loosely generalizing Stone duality, maintains that the category of 'point-free spaces' is the opposite of the category of frames (that is, complete lattices in which the meet distributes over arbitrary joins). Earlier work by the first three authors shows how a noncommutative $\mathrm{C}^{*}$-algebra gives rise to a commutative one internal to a certain sheaf topos. The latter, then, has a constructive Gelfand spectrum, also internal to the topos in question. After a brief review of this work, we compute the so-called external description of this internal spectrum, which in principle is a fibred point-free space in the familiar topos of sets and functions. However, we obtain the external spectrum as a fibred topological space in the usual sense. This leads to an explicit Gelfand transform, as well as to a topological reinterpretation of the Kochen-Specker theorem of quantum mechanics.
\end{abstract}

2010 Mathematics subject classification: primary 46L85; secondary 18B25, 47L40, 06D22, 03 F55.

Keywords and phrases: Gelfand spectrum, noncommutative $\mathrm{C}^{*}$-algebra, topos, locale.

\section{Generalized spaces}

Gelfand duality is the categorical equivalence

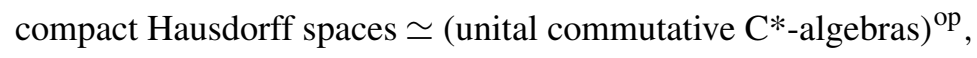

where the choice of arrows in both categories is implicit (but obvious, that is, continuous maps and unital *-homomorphisms, respectively). For simplicity, we restrict ourselves to the compact/unital case. Furthermore, given a category $\mathrm{C}$, the opposite category $\mathrm{C}^{\mathrm{op}}$ has the same objects as $\mathrm{C}$, but has all arrows reversed. The

Heunen was supported by the Netherlands Organisation for Scientific Research through a Rubicon grant; Spitters was supported by the Netherlands Organisation for Scientific Research through the DIAMANT cluster; Wolters was supported by the Netherlands Organisation for Scientific Research through project 613.000 .811 .

(C) 2011 Australian Mathematical Publishing Association Inc. 1446-7887/2011 \$16.00 
functors implementing the equivalence (1) are, of course, $C: X \mapsto C(X) \equiv C(X, \mathbb{C})$ from left to right, with pullback on arrows, and $\Sigma: A \mapsto \Sigma(A)$ from right to left, where $\Sigma(A)$ is the Gelfand spectrum of $A$ (realized, for example, as the space of unital multiplicative linear maps $A \rightarrow \mathbb{C}$ equipped with the relative weak*-topology), and similarly pullback on arrows.

Subsequently, there are (at least) two possible directions to take.

First, the modern approach is to take the quantum jump of defining the category of 'noncommutative spaces' up to equivalence by

$$
\text { noncommutative spaces } \simeq\left(\mathrm{C}^{*} \text {-algebras }\right)^{\mathrm{op}} \text {. }
$$

Here a major surprise arises, which is quite unexpected from the categorical setting: according to the (second) Gelfand-Naimark theorem, a noncommutative space acts as an operator algebra on some Hilbert space. It is the combination of this Hilbert space setting deriving from the right-hand side of (2) and the call for geometrical and topological techniques - adapted to the noncommutative setting-coming from the left-hand side that gives noncommutative geometry its strength $[10,11]$.

Second, and more traditionally, one may attempt to generalize the notion of Gelfand duality to noncommutative $\mathrm{C}^{*}$-algebras $A$. There have been many such attempts, which may be grouped according to the specific notion of a Gelfand spectrum that is used. For example, in the Dauns-Hofmann theorem [14, 15, 29] the Gelfand spectrum of $A$ is taken to be the Gelfand spectrum of its centre $Z(A)$, on which $A$ is realized as a sheaf. Akemann, on the other hand, used the space of maximal left ideals of $A$, but needed to generalize the notions of topology and continuity [1]. Shultz used the pure state space of $A$, equipped with the structure of a transition probability [31], later refined so as to make the noncommutative Gelfand spectrum a so-called Poisson space with a transition probability [25, 26]. See also [9, 24]. In all cases, the point is to realize $A$ in a way that resembles a space of complex-valued continuous functions as much as possible.

Ultimately, what lies behind both directions is the success of Gelfand duality in capturing (compact Hausdorff) spaces algebraically. What is slightly unnatural, though, is that this capturing should involve the complex (or, for that matter, the real) numbers in a fundamental way. This may be avoided in an order-theoretic approach, as follows [19], [28, Ch. IX]. Instead of the passage $X \mapsto C(X)$ from spaces to complex algebras, we take $X \mapsto \mathcal{O}(X)$, where $\mathcal{O}(X)$ is just the topology of $X$ in the defining sense of its collection of open sets. This has a natural lattice structure under inclusion, and in fact defines a highly structured kind of lattice known as a frame. This is a complete distributive lattice such that $x \wedge \bigvee_{\lambda} y_{\lambda}=\bigvee_{\lambda}\left(x \wedge y_{\lambda}\right)$ for arbitrary families $\left\{y_{\lambda}\right\}$ (and not just for finite ones, in which case the said property follows from the definition of a distributive lattice). Indeed, $\mathcal{O}(X)$ is a frame with $U \leq V$ if $U \subseteq V$. A frame homomorphism preserves finite meets and arbitrary joins; this leads to the category of frames and frame homomorphisms.

In order to have an equivalence like (1), we need to cut down both the category of spaces and the category of frames. To do so, we first define a point of a frame $F$ 
as a frame map $p^{*}: F \rightarrow\{0,1\}$, where as a frame $\{0,1\}$ is identified with $\mathcal{O}(*)$, that is, the topology of a space with a point (so that we identify 0 with $\emptyset$ and 1 with $*$ ). In fact, if $F=\mathcal{O}(X)$, then any point $p \in X$ defines a point of $F$ by $p^{*}=p^{-1}$ (that is, $p^{*}(U)=1$ if and only if $\left.p \in U\right)$. Using this concept, the $\operatorname{set} \operatorname{Pt}(F)$ of points of a frame $F$ may be topologized in a natural way, by declaring its open sets to be the sets of the form $\operatorname{Pt}(U)=\left\{p^{*} \in \operatorname{Pt}(F) \mid p^{*}(U)=1\right\}$, where $U \in F$. We say that a frame $F$ is spatial if it is isomorphic to $\mathcal{O}(\operatorname{Pt}(F))$ in the category of frames. On the other hand, a topological space $X$ is called sober if it is homeomorphic to $\operatorname{Pt}(\mathcal{O}(X))$. Given these definitions, it is almost tautological that

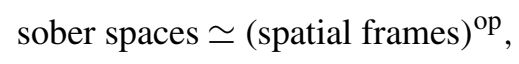

where the equivalence is given by $\mathcal{O}$ and $\mathrm{Pt}$ (seen as functors). Though (3) is true almost by definition, the nontrivial statement of Stone duality, that is,

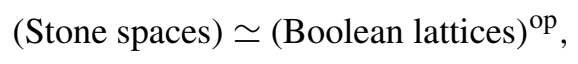

is actually a special case of (3). The nontrivial observation-apart from the fact that Hausdorffness implies soberness-is that although Stone spaces form a subcategory of sober spaces, Boolean lattices are not a subcategory of frames (for one thing, a Boolean lattice need not be complete). Hence a special manoeuvre is needed to embed Boolean lattices in frames, which is done through the so-called ideal completion $L \mapsto \operatorname{Idl}(L)$; this is the collection of nonempty lower closed subsets $I \subset L$ such that $x, y \in I$ implies $x \vee y \in I$, ordered by inclusion [19, p. 59]. A Stone space $X$ then defines the Boolean lattice $\mathcal{O}_{c}(X)$ of closed and open subsets of $X$, whose ideal completion is the topology $\mathcal{O}(X)$; conversely, a Boolean lattice $L$ defines a Stone space $X=\operatorname{Pt}(\operatorname{Idl}(L))$, with $\mathcal{O}(X) \cong \operatorname{Idl}(L)$.

Let us note the following, however. It is easily shown that a frame $F$ is spatial if and only if $F \cong \mathcal{O}(X)$ for some space $X$, not necessarily sober-in fact, we will later encounter an example of exactly this situation. In that case, following [19], we may call

$$
X^{S}=\operatorname{Pt}(\mathcal{O}(X))
$$

which is necessarily sober, the soberification of $X$ (if $X$ is already sober, one has $X^{S} \cong X$ ). This construction may be compared to the passage from a compact nonHausdorff space $X$ to its Hausdorffication

$$
X^{H}=\Sigma(C(X)) .
$$

Now recall that the step from (1)-(2) introduced a certain generalization of the concept of space by omitting the qualifier 'unital commutative' in the characterization of spaces in the right-hand side of (1). Analogously, we may omit the qualifier 'spatial' in the right-hand side of (3), hoping to arrive at a different generalized notion of space. Following [19, 22, 28], we therefore write

$$
\text { point-free spaces } \simeq(\text { frames })^{\mathrm{op}},
$$


which, like (2), is no longer a duality theorem, but a statement of the definition of the category of 'point-free spaces' (also known as locales). This definition comes with a curious piece of notation: any frame is written $\mathcal{O}(X)$, whether or not it is spatial, and the corresponding point-free space is written as $X$. Furthermore, the symbol $C(X, Y)$ denotes the object (in whatever category the frames are defined) of frame maps from $\mathcal{O}(Y)$ to $\mathcal{O}(X)$; a 'continuous' map $f: X \rightarrow Y$ is nothing but a frame map from $\mathcal{O}(Y)$ to $\mathcal{O}(X)$, which tends to be written as $f^{*}$ or $f^{-1}$. This notation is partly motivated by the case where $\mathcal{O}(X)$ are $\mathcal{O}(Y)$ actually the topologies of sober spaces $X$ and $Y$, respectively, for in that case it can be shown (nonconstructively) that any frame map $f^{*}: \mathcal{O}(Y) \rightarrow \mathcal{O}(X)$ is of the form $f^{*}=f^{-1}$ for a continuous map $f: X \rightarrow Y$ in the usual sense.

The surprising role of Hilbert spaces in the theory of noncommutative spaces has a counterpart for point-free spaces: these turn out to be related to logic, especially to intuitionistic propositional logic. (Perhaps this is less surprising in view of Stone duality and the well-known connection between Boolean lattices and classical propositional logic.) Indeed, a frame is a complete Heyting algebra, where a Heyting algebra is a distributive lattice $\mathcal{L}$ with a map $\rightarrow: \mathcal{L} \times \mathcal{L} \rightarrow \mathcal{L}$ satisfying $x \leq(y \rightarrow z)$ if and only if $x \wedge y \leq z$, called implication [17, 28, 34]. Unlike in a Boolean lattice, negation is now a derived notion, defined by $\neg x=(x \rightarrow \perp)$.

Every Boolean algebra is a Heyting algebra, but not vice versa; in fact, a Heyting algebra is Boolean if and only if $\neg \neg x=x$ for all $x$, which is the case if and only if $\neg x \vee x=\top$ for all $x$; not necessarily granting this is the essence of intuitionistic logic. The point, then, is that a complete Heyting algebra is essentially the same thing as a frame, for in a frame one may define $y \rightarrow z=\bigvee\{x \mid x \wedge y \leq z\}$, and conversely, the infinite distributivity law in a frame is automatically satisfied in a Heyting algebra.

In principle, noncommutative spaces and point-free spaces (that is, locales) appear to be totally different generalizations of the notion of a topological space. However, a close connection arises if we return to Gelfand duality. To explain this, note that the usual proofs of Gelfand duality are nonconstructive; for example, if the Gelfand spectrum is realized as the maximal ideal space of $A$, one needs Zorn's lemma. However, a typical situation in constructive mathematics now arises: Gelfand duality is nonconstructively equivalent to a result that is constructively valid (that is, provable without using the axiom of choice or the exclusion of the middle third) $[2-4,12,13]$. Hence the constructive version of the key ingredient of classical Gelfand duality, namely the isomorphism

$$
A \cong C(\Sigma(A), \mathbb{C})
$$

of commutative $\mathrm{C}^{*}$-algebras, is formally the very same statement, but now reinterpreted according to the notation for frame maps just explained. Thus the Gelfand spectrum $\Sigma(A)$ and the complex numbers $\mathbb{C}$ are now objects of the category of point-free spaces, that is, they are really frames $\mathcal{O}(\Sigma(A))$ and $\mathcal{O}(\mathbb{C})$, which are not necessarily spatial, and $C(\Sigma(A), \mathbb{C})$ denotes the object (in the ambient category) of frame maps from $\mathcal{O}(\mathbb{C})$ to $\mathcal{O}(\Sigma(A))$. (Technically, $\mathcal{O}(\Sigma(A))$ is required to be 
compact and completely regular [4], which are frame-theoretic properties replacing the combination compact Hausdorff for topological spaces [19].)

The choice between the constructive version of Gelfand duality (in terms of pointfree spaces) and its familiar nonconstructive counterpart (in terms of topological spaces) is not a matter of philosophical taste. In set theory, the usual version is perfectly acceptable to us. The point is that constructive Gelfand duality holds in arbitrary topoi (with natural numbers objects, so that internal $\mathrm{C}^{*}$-algebras can be defined). We refer to $[20,21]$ for an encyclopaedic treatment of topos theory, to [6, 17, 28] for (complementary) book-length introductions, to [34] for a shorter treatment, and finally to the appendix of [8] for a very brief survey of exactly what is needed below. The notion of a $\mathrm{C}^{*}$-algebra in a topos with natural numbers object, including the statement and proof of Gelfand duality in the commutative case, is due to Banaschewski and Mulvey [4]. See also [18] for a review of this theory, including a reformulation along the lines of $[12,13]$.

\section{Internal Gelfand spectrum}

In order to define Gelfand spectra for noncommutative $\mathrm{C}^{*}$-algebras, we proceed as follows [18]. Let $A$ be a unital $\mathrm{C}^{*}$-algebra, and let $\mathcal{C}(A)$ be the poset of unital commutative $\mathrm{C}^{*}$-subalgebras of $A$ (ordered by set-theoretic inclusion), equipped with the Alexandrov topology. (The open sets $U$ of the Alexandrov topology on a poset $P$ are the upward closed sets (if $x \in U$ and $x \leq y$, then $y \in U$ ). The sets $U_{x}=\uparrow x=$ $\{y \in P \mid y \geq x\}, x \in P$, form a basis of the Alexandrov topology.) Thus we have the topos $\operatorname{Sh}(\mathcal{C}(A))$ of sheaves on $\mathcal{C}(A)$. We now define a specific sheaf $\underline{A}$ on $\mathcal{C}(A)$ by

$$
\underline{A}(\uparrow C)=C, \quad \forall C \in \mathcal{C}(A) .
$$

This formula defines $\underline{A}$ on the basic open sets $U_{C}=\uparrow C$ of $\mathcal{C}(A)$ in the Alexandrov topology. On an arbitrary open set $U=\bigcup_{C \in \Gamma} U_{C}$, the sheaf property gives $\underline{A}(U)=$ $\lim _{C \in \Gamma} \underline{A}\left(U_{C}\right)$. Under the identification of $\operatorname{Sh}(P)$ with Sets ${ }^{P}$ (where the poset $P$ is seen as a category in the usual way) through the correspondence $F(\uparrow x) \leftrightarrow F(x)$ [17], the sheaf $\underline{A}$ corresponds to the tautological functor $C \mapsto C$ in $\operatorname{Sets}^{\mathcal{C}(A)}$. If $C \subseteq D$, then $\uparrow D \subseteq \uparrow C$, and the map $\underline{A}(\uparrow C) \rightarrow \underline{A}(\uparrow D)$, that is, $C \rightarrow D$, is simply given by inclusion. This sheaf turns out to be a commutative $\mathrm{C}^{*}$-algebra $\underline{A}$ in $\operatorname{Sh}(\mathcal{C}(A))$ under natural operations, so that it has an internal Gelfand spectrum $\underline{\Sigma}(\underline{A})$. With $A$ fixed, we will henceforth simply call this spectrum $\underline{\Sigma}$; it is a point-free space in the topos $\operatorname{Sh}(\mathcal{C}(A))$. The functorial properties of the map $A \mapsto \underline{\Sigma}(\underline{A})$, as well as of the map $A \mapsto \Sigma(A)$ to be introduced below, have been studied in [33].

The explicit computation of $\underline{\Sigma}$ was initiated in [18], and was completed in [35]. To state the result (that is, Theorem 1 below), topologize the disjoint union

$$
\Sigma=\coprod_{C \in \mathcal{C}(A)} \Sigma(C),
$$


where $\Sigma(C)$ is the usual Gelfand spectrum of $C \in \mathcal{C}(A)$ (that is, the set of pure states or characters on $C$ with the relative weak*-topology) by saying that $\mathcal{U} \in \mathcal{O}(\Sigma)$ if and only if the following two conditions are satisfied for all $C \in \mathcal{C}(A)$ (with the notation $\left.\mathcal{U}_{C} \equiv \mathcal{U} \cap \Sigma(C)\right)$.

(1) $\mathcal{U}_{C} \in \mathcal{O}(\Sigma(C))$.

(2) For all $D \supseteq C$, if $\lambda \in \mathcal{U}_{C}$ and $\lambda^{\prime} \in \Sigma(D)$ such that $\lambda_{\mid C}^{\prime}=\lambda$, then $\lambda^{\prime} \in \mathcal{U}_{D}$.

For each $U \in \mathcal{O}(\mathcal{C}(A))$, we also introduce the space

$$
\Sigma_{U}=\coprod_{C \in U} \Sigma(C)
$$

with relative topology inherited from $\Sigma$. Then we obtain the following theorem.

Theorem 1. Let $A$ be a unital $C^{*}$-algebra A. The frame $\mathcal{O}(\underline{\Sigma})$ in $\operatorname{Sh}(\mathcal{C}(A))$ that underlies the internal Gelfand spectrum $\underline{\Sigma} \equiv \underline{\Sigma}(\underline{A})$ of the internal commutative $C^{*}$ algebra $\underline{A}$ defined by (8) is given by the sheaf

$$
\mathcal{O}(\underline{\Sigma}): U \mapsto \mathcal{O}\left(\Sigma_{U}\right)
$$

where $U \in \mathcal{O}(\mathcal{C}(A))$; if $U \subseteq V$, the map $\mathcal{O}\left(\Sigma_{V}\right) \rightarrow \mathcal{O}\left(\Sigma_{U}\right)$ is given by $\mathcal{U} \mapsto \mathcal{U} \cap \Sigma_{U}$.

The proof of this theorem is rather lengthy, requiring familiarity with constructive mathematics, as well as with the closely related technique of internal reasoning in topos theory. Besides the general theory of internal Gelfand duality in Grothendieck topoi due to Banaschewski and Mulvey [4] looming in the background, the proof of Theorem 1 consist of three main steps:

(1) the lattice-theoretic description of general constructive Gelfand spectra [12, 13];

(2) the specific application of this description to the commutative $\mathrm{C}^{*}$-algebra $\underline{A}$ in the topos $\operatorname{Sh}(\mathcal{C}(A))[18]$;

(3) the insight that this application yields the explicit form (11) [32, 35].

We now give a summary of these steps, referring to the papers just cited for further details. In fact, the third step can be carried out in two rather different ways, of which the approach of [35] is easier to explain to operator algebraists. Hence in what follows we use the latter. The techniques in [32] will be further explored in future work in collaboration with Vickers, whom we wish to thank for his insightful comments on an earlier version of this paper. In what follows, $A$ is a commutative $\mathrm{C}^{*}$-algebra with unit in some topos (with natural numbers object), while $C$ is a commutative $\mathrm{C}^{*}$-algebra with unit in the usual sense, that is, in the topos Sets of sets and functions.

Step 1. As already mentioned, the constructive approach to Gelfand duality emphasizes the frame $\mathcal{O}(\Sigma)$ rather than the set $\Sigma \equiv \Sigma(A)$. To construct $\mathcal{O}(\Sigma)$, take the usual positive cone $A^{+}:=\left\{a \in A_{\text {sa }} \mid a \geq 0\right\}$ of $A$ (where $A_{\text {sa }}$ is the selfadjoint part of $A$ ), and define $a \preccurlyeq b$ if and only if there exists $n \in \mathbb{N}$ such that $a \leq n b$. Define $a \approx b$ if and only if $a \preccurlyeq b$ and $b \preccurlyeq a$. The lattice operations on $A_{\text {sa }}$ (defined with respect to the usual partial order $\leq$ ) respect $\approx$ and hence $L_{A}=A^{+} / \approx$ is a lattice under the descent of $\leq$ to the quotient, which we denote by $\leq$. 
If $A$ is finite-dimensional, the constructive Gelfand spectrum of $A$ is simply (isomorphic to) the ideal completion $\operatorname{Idl}\left(L_{A}\right)$ of $L_{A}$ (see the paragraph following (3)). In general, one needs to refine this construction. First, define a surjective map $A_{\mathrm{sa}} \rightarrow L_{A}$, $a \mapsto \mathrm{D}_{a} \equiv\left[a^{+}\right]$, where $a=a^{+}-a^{-}, a^{ \pm} \in A^{+}$, and $\left[a^{+}\right]$is the equivalence class of $a^{+}$in $L_{A}$ with respect to $\approx$. Second, write $\mathrm{D}_{b} \ll \mathrm{D}_{a}$ if and only if $\mathrm{D}_{b} \leq \mathrm{D}_{a-q}$ for some $q>0, q \in \mathbb{Q}$. Third, we refine the down-set $\downarrow \mathrm{D}_{a}=\left\{\mathrm{D}_{b} \in L_{A} \mid \mathrm{D}_{b} \leq \mathrm{D}_{a}\right\}$ to $\downarrow \mathrm{D}_{a}=\left\{\mathrm{D}_{b} \in L_{A} \mid \mathrm{D}_{b} \ll \mathrm{D}_{a}\right\}$, and declare an ideal $I \in \operatorname{Idl}\left(L_{A}\right)$ to be regular if $I \supseteq \Downarrow \mathrm{D}_{a}$ for some $\mathrm{D}_{a} \in L_{A}$ implies $\mathrm{D}_{a} \in I$ (in other words, if $\mathrm{D}_{b} \in I$ for all $\mathrm{D}_{b} \ll \mathrm{D}_{a}$, then $\left.\mathrm{D}_{a} \in I\right)$. This yields the frame $\operatorname{RIdl}\left(L_{A}\right)$ of regular ideals of $L_{A}$, ordered by inclusion (like $\operatorname{Idl}\left(L_{A}\right)$, of which $\operatorname{RIdl}\left(L_{A}\right)$ is a subframe). The constructive Gelfand spectrum of $A$, then, turns out to be (isomorphic to) just this subframe, that is,

$$
\mathcal{O}(\Sigma(A)) \cong \operatorname{RIdl}\left(L_{A}\right) .
$$

There is a natural map $\tilde{f}_{A}: L_{A} \rightarrow \operatorname{Idl}\left(L_{A}\right), \mathrm{D}_{a} \mapsto \downarrow \mathrm{D}_{a}$, which may be refined to a map $f_{A}: L_{A} \rightarrow \operatorname{RIdl}\left(L_{A}\right)$ that sends $\mathrm{D}_{a}$ to the smallest regular ideal containing $\tilde{f}_{A}\left(\mathrm{D}_{a}\right)=\downarrow \mathrm{D}_{a}$; explicitly, one has

$$
f_{A}\left(\mathrm{D}_{a}\right)=\left\{\mathrm{D}_{c} \in L_{A} \mid \mathrm{D}_{b} \ll \mathrm{D}_{c} \Rightarrow \mathrm{D}_{b} \leq \mathrm{D}_{a}, \mathrm{D}_{b} \in L_{A}\right\} .
$$

If one thinks of $\mathcal{O}(\Sigma)$ as the 'topology' of the Gelfand spectrum (in the appropriate point-free sense), the 'open sets' $f_{A}\left(\mathrm{D}_{a}\right)$ (or, less accurately, the elements $\mathrm{D}_{a}$ of $L_{A}$ themselves), comprise 'basic open sets' for the topology, in terms of which general 'open sets' $U \in \operatorname{RIdl}\left(L_{A}\right)$ may be expressed as

$$
U=\bigvee\left\{f_{A}\left(\mathrm{D}_{a}\right) \mid \mathrm{D}_{a} \in L_{A}, f_{A}\left(\mathrm{D}_{a}\right) \leq U\right\} .
$$

Applying this to ordinary unital commutative $\mathrm{C}^{*}$-algebras $C$, one finds that the frame $\mathcal{O}(\Sigma)$ is spatial, being related to the usual Gelfand topology $\mathcal{O}(\Sigma(C))$ by the frame isomorphism $\operatorname{RIdl}\left(L_{C}\right) \rightarrow \mathcal{O}(\Sigma(C))$ that on basic open sets is given by

$$
f_{C}\left(\mathrm{D}_{a}\right) \mapsto \mathcal{D}_{a} \equiv\{\varphi \in \Sigma(C) \mid \varphi(a)>0\} \quad \forall a \in C_{\mathrm{sa}} .
$$

In particular, the map

$$
\mathrm{f}_{C}: L_{C} \rightarrow \mathcal{O}(\Sigma(C)), \quad \mathrm{D}_{a} \mapsto \mathcal{D}_{a}
$$

is well defined (that is, independent of the choice of $a$ ); see [35, Lemma 2.14].

Step 2. Internalizing the above construction of $\mathcal{O}(\Sigma)$ to the topos $\operatorname{Sh}(\mathcal{C}(A))$ and applying it to the internal C*-algebra $\underline{A}$ yields a lattice $\underline{L}_{A}$ in $\operatorname{Sh}(\mathcal{C}(A))$, given by [18, Theorem 20]

$$
\underline{L}_{\underline{A}}(\uparrow C)=L_{C} .
$$

Interpreting RIdl in the topos $\operatorname{Sh}(\mathcal{C}(A))$ with Kripke-Joyal semantics [28] shows that the internal frame $\underline{\operatorname{RIdl}}\left(\underline{L}_{A}\right)$ in $\operatorname{Sh}(\mathcal{C}(A))$ is given by the sheaf (see [18, Theorem 29])

$$
\underline{\operatorname{RIdl}}\left(\underline{L}_{A}\right): U \mapsto\left\{\underline{F} \in \operatorname{Sub}\left(\underline{L}_{A} \mid U\right) \mid \underline{F}(\uparrow C) \in \operatorname{RIdl}\left(L_{C}\right) \text { for all } C \in U\right\} .
$$


Here $\underline{L}_{A \mid U}: \mathcal{O}(U)^{\mathrm{op}} \rightarrow$ Sets is the restriction of the sheaf $\underline{L}_{A}: \mathcal{O}(\mathcal{C}(A))^{\mathrm{op}} \rightarrow$ Sets to $\mathcal{O}(U)$, where $U \in \mathcal{O}(\mathcal{C}(A))$, and $\operatorname{Sub}\left(\underline{L}_{A \mid U}\right)$ is the set of subsheaves of $\underline{L}_{A \mid U}$; note that $\underline{F}(\uparrow C) \subseteq L_{C}$ by (14), so $\underline{F}(\uparrow C) \in \overline{\operatorname{RIdl}}\left(L_{C}\right)$ in (15) is well defined. If $U \subseteq V$, then the map $\underline{\operatorname{RIdl}}\left(\underline{L}_{A}\right)(V) \rightarrow \underline{\operatorname{RIdl}}\left(\underline{L}_{A}\right)(U)$ is given by restricting $\underline{F} \in \operatorname{Sub}\left(\underline{L}_{\underline{A} \mid V}\right)$ to $\mathcal{O}(U)$.

Step 3. To prove (15), it can be shown that the transformation $\theta: \underline{\operatorname{RIdl}}\left(\underline{L}_{A}\right) \rightarrow \mathcal{O}(\underline{\Sigma})$, defined by its components

$$
\begin{aligned}
\theta_{U}:\left\{\underline{F} \in \operatorname{Sub}\left(\underline{L}_{A} \mid U\right) \mid\right. & \left.\underline{F}(\uparrow C) \in \operatorname{RIdl}\left(L_{C}\right) \text { for all } C \in U\right\} \rightarrow \mathcal{O}\left(\Sigma_{U}\right), \\
\underline{F} & \mapsto \coprod_{C \in U} \bigcup_{D_{a} \in \underline{F}(\uparrow C)} \mathcal{D}_{a},
\end{aligned}
$$

is a natural isomorphism (since $\underline{\operatorname{RIdl}}\left(\underline{L}_{A}\right)$ and $\mathcal{O}(\underline{\Sigma})$ are internal frames, it suffices to prove that $\theta_{\mathcal{C}(A)}$ is an isomorphism of frames in Sets; see [35, Theorem 2.17]). Note that $\theta_{U}(\underline{F})$ indeed lies in $\mathcal{O}\left(\Sigma_{U}\right)$ by the property $\rho_{D C}^{-1} \circ \mathrm{f}_{C}=\mathrm{f}_{D} \circ \iota_{C D}$ for all $C, D \in \mathcal{C}(A)$ such that $C \subseteq D$, where $\rho_{D C}^{-1}: \mathcal{O}(\Sigma(C)) \rightarrow \mathcal{O}(\Sigma(D))$ is the inverse image map of the restriction $\rho_{D C}: \Sigma(D) \rightarrow \Sigma(C), \lambda \mapsto \lambda_{\mid C}$, and $\iota_{C D}: L_{C} \rightarrow L_{D}$ is the obvious embedding $\mathrm{D}_{a} \mapsto \mathrm{D}_{a}$ (where $a \in C$ in the first $\mathrm{D}_{a}$ and $a \in D$ in the second).

We illustrate Theorem 1 when $A=M_{n}(\mathbb{C})$, the set of $n \times n$ complex matrices. We then have a frame isomorphism $\mathcal{O}(\Sigma(C)) \cong \mathcal{P}(C)$ for any $C \in \mathcal{C}(A)$ [8], where $\mathcal{P}(C)$ is the projection lattice of $C$ (and similarly, $\mathcal{P}(A)$ below is the projection lattice of $A$ ). Hence

$$
\mathcal{O}(\Sigma) \cong\{S: \mathcal{C}(A) \rightarrow \mathcal{P}(A) \mid S(C) \in \mathcal{P}(C), S(C) \leq S(D) \text { if } C \subseteq D\},
$$

where the right-hand side is equipped with the pointwise partial order $\leq$ with respect to the usual partial ordering $\leq$ of projections, that is, $S \leq T$ if and only if $S(C) \leq T(C)$ for all $C \in \mathcal{C}(A)$. To obtain (17) we identify $\mathcal{U}=\bigsqcup_{C \in \mathcal{C}(A)} \mathcal{U}_{C}$ as an element of $\mathcal{O}(\Sigma)$ with $S: \mathcal{C}(A) \rightarrow \mathcal{P}(A)$ on the right-hand side of $(17)$, where $S(C) \in \mathcal{P}(C)$ is the image of $\mathcal{U}_{C} \in \mathcal{O}(\Sigma(C))$ under the isomorphism $\mathcal{O}(\Sigma(C)) \rightarrow \mathcal{P}(C)$ just mentioned. Similarly, for $U \in \mathcal{O}(\mathcal{C}(A))$, the frame $\mathcal{O}\left(\Sigma_{U}\right)$ may be identified with a collection of maps $S: U \rightarrow \mathcal{P}(A)$ satisfying the conditions in (17).

\section{External Gelfand spectrum}

It is not so easy for $\mathrm{C}^{*}$-algebraists to deal with point-free spaces in a sheaf topos $\mathrm{Sh}(X)$. Fortunately, such spaces have a so-called external description in ordinary set theory $[16,21,22]$. In fact, a point-free space $\underline{Y}$ in $\operatorname{Sh}(X)$ may be represented by a continuous map $\pi: Y \rightarrow X$, where $Y$ is a point-free space in the usual sense (that is, in Sets), with frame $\mathcal{O}(Y)=\mathcal{O}(\underline{Y})(X)$; here $\mathcal{O}(\underline{Y})$ is the internal frame in $\operatorname{Sh}(X)$ associated to $\underline{Y}$. The reader will now have become used to the idea that the notation $\pi: Y \rightarrow X$ really denotes a frame map $\pi^{*}: \mathcal{O}(X) \rightarrow \mathcal{O}(Y)$, nothing being 
implied about the possible spatiality of the frames in question. In terms of $\pi^{*}$, one may reconstruct $\underline{Y}$ from $\pi: Y \rightarrow X$ as the sheaf

$$
\mathcal{O}(\underline{Y}): U \mapsto\left\{V \in \mathcal{O}(Y) \mid V \leq \pi^{*}(U)\right\} \quad \forall U \in \mathcal{O}(X) .
$$

Furthermore, if $\underline{Y}_{1}$ and $\underline{Y}_{2}$ are two point-free spaces in $\operatorname{Sh}(X)$, with external descriptions $\pi_{i}: Y_{i} \rightarrow X, i=1,2$, then an internal continuous map $\underline{f}: \underline{Y}_{1} \rightarrow \underline{Y}_{2}$ is given externally by a continuous map $f: Y_{1} \rightarrow Y_{2}$ satisfying $\pi_{2} \circ f=\pi_{1}$.

Applying this to $X=\mathcal{C}(A)$ and $Y=\Sigma$ we obtain the following result.

THEOREM 2. The external description of the point-free Gelfand spectrum $\underline{\Sigma}$ may be identified with the canonical projection

$$
\pi: \Sigma \rightarrow \mathcal{C}(A)
$$

where $\Sigma$ is seen as an ordinary (rather than a point-free) topological space, as is $\mathcal{C}(A)$.

We remark that $\pi$ is the canonical projection in the sense that, if $\sigma \in \Sigma(C) \subset \Sigma$, then $\pi(\sigma)=C$. From this point of view, $\mathcal{O}(\Sigma)$ is actually the weakest topology making this projection continuous with respect to the Alexandrov topology on $\mathcal{C}(A)$.

PROOF. Taking $X=\mathcal{C}(A)$ and $\underline{Y}=\underline{\Sigma}$, we see from (11) that $\mathcal{O}(\underline{\Sigma})(\mathcal{C}(A))=\mathcal{O}(\Sigma)$, which frame is obviously spatial. To be precise, in point-free topology a notation like (19) is typically used for a map between point-free spaces, which by definition is the frame map $\pi^{-1}: \mathcal{O}(\mathcal{C}(A)) \rightarrow \mathcal{O}(\Sigma)$. In this case, however, the frame map $\pi^{-1}$ is actually the inverse image map of the continuous map (19), interpreted in the usual topological way.

Conversely, from (18) and (19) we immediately recover (11).

Theorem 2 has a number of interesting applications. We first turn to the Gelfand transform. Unlike other approaches to Gelfand duality for noncommutative $\mathrm{C}^{*}$ algebras, our aim is not to reconstruct $A$, but rather its 'Bohrification' $\underline{A}$, since it is the latter that carries the physical content of $A$, at least according to Bohr's 'doctrine of classical concepts' [5] as reformulated mathematically in [27].

The Gelfand isomorphism (7) holds internally in $\operatorname{Sh}(\mathcal{C}(A))$, that is, one has

$$
\underline{A} \cong C(\underline{\Sigma}, \underline{\mathbb{C}})
$$

as an isomorphism of sheaves respecting the $\mathrm{C}^{*}$-algebraic structure on both sides. (Recall that isomorphisms of sheaves in sheaf topoi are simply natural isomorphisms of functors [28].) Here $\underline{\mathbb{C}}$ is the point-free space of complex numbers in $\operatorname{Sh}(\mathcal{C}(A))$ with associated frame $\mathcal{O}(\mathbb{C})$ (not to be confused with the complex numbers object in $\operatorname{Sh}(\mathcal{C}(A))$, given by the sheaf $U \mapsto C(U, \mathbb{C}))$, defined by the sheaf

$$
\mathcal{O}(\underline{\mathbb{C}}): U \mapsto \mathcal{O}(U \times \mathbb{C}) \quad \forall U \in \mathcal{O}(\mathcal{C}(A)) .
$$

It follows from $[8$, Section 5, (5.12)] and (11) that as a sheaf one has

$$
C(\underline{\Sigma}, \underline{\mathbb{C}}): U \mapsto C\left(\Sigma_{U}, \mathbb{C}\right),
$$


where $\Sigma_{U}=\bigsqcup_{C \in U} \Sigma(C)$; if $U \subseteq V$, the map $C\left(\Sigma_{V}, \mathbb{C}\right) \rightarrow C\left(\Sigma_{U}, \mathbb{C}\right)$ is given by the pullback of the inclusion $\Sigma_{U} \hookrightarrow \Sigma_{V}$ (that is, by restriction). It then follows from (8) and (22) that the isomorphism (20) is given by its components

$$
\underline{A}(U) \cong C\left(\Sigma_{U}, \mathbb{C}\right) \text {. }
$$

In particular, the component of the natural isomorphism in (20) at $U=\uparrow C$ is

$$
C \cong C\left(\Sigma_{\uparrow C}, \mathbb{C}\right) .
$$

A glance at the topology of $\Sigma$ shows that the Hausdorffication (5) is given by $\Sigma_{\uparrow C}^{H} \cong$ $\Sigma(C)$, so that the isomorphism (24) comes down to the usual Gelfand isomorphism

$$
C \cong C\left(\Sigma_{C}, \mathbb{C}\right) \text {. }
$$

At the end of the day, the Gelfand isomorphism (20) therefore turns out to simply assemble all isomorphisms (25) for the commutative $\mathrm{C}^{*}$-subalgebras $C$ of $A$ into a single sheaf-theoretic construction. Incidentally, taking $C=\mathbb{C} \cdot 1$ in (24) shows that $\Sigma^{H}$ is a point, which is also obvious from the fact that any open set containing the point $\Sigma(\mathbb{C} \cdot 1)$ of $\Sigma$ must be all of $\Sigma$.

Second, we give a topological reinterpretation of the celebrated Kochen-Specker theorem [23]. It was the sheaf-theoretic reformulation of the Kochen-Specker theorem by Butterfield and Isham [7] that originally got the the use of topos theory in the foundations of quantum physics going. What follows is a simplification of [8, Section 6], which was written when the spatial nature of $\Sigma$ was not yet understood. See also [18, Theorem 6] for an internal proof of the equivalence between the first two bullet points below.

We say that a valuation on a $\mathrm{C}^{*}$-algebra $A$ is a nonzero map $\lambda: A_{\mathrm{sa}} \rightarrow \mathbb{R}$ that is linear on commuting operators and dispersion-free, that is, $\lambda\left(a^{2}\right)=\lambda(a)^{2}$ for all $a \in A_{\mathrm{sa}}$. If $A$ is commutative, the Gelfand spectrum $\Sigma(A)$ consists precisely of the valuations on $A$. Physically, a valuation corresponds to a so-called noncontextual hidden variable, which assigns a sharp value to each observable a per se. A contextual hidden variable gives a sharp value to a seen in a specific measurement context in which it, in particular, may be measured. See, for example, [30]. In our mathematization, measurement contexts are identified with commutative $\mathrm{C}^{*_{-}}$ subalgebras of some ambient noncommutative $\mathrm{C}^{*}$-algebra $A$, so that a contextual hidden variable assigns a value to a pair $(a, C)$ where $a \in C$. Hence Theorem 3 below identifies noncontextual hidden variables with continuous cross-sections of $\pi: \Sigma \rightarrow \mathcal{C}(A)$, and contextual hidden variables correspond to possibly discontinuous cross-sections.

The mathematics neatly fits the physics here, but it should be realized that specific examples of $\mathrm{C}^{*}$-algebras $A$ may suggest coarser natural topologies on $\mathcal{C}(A)$ than the Alexandrov topology (like the Scott topology), which in turn may imply stronger continuity conditions. We thank the referee for this comment.

THEOREM 3. There is a bijective correspondence between the following:

- valuations on A; 
- $\quad$ points of $\underline{\Sigma}(\underline{A})$ in $\operatorname{Sh}(\mathcal{C}(A))$;

- $\quad$ continuous sections $\sigma: \mathcal{C}(A) \rightarrow \Sigma$ of the bundle $\pi: \Sigma \rightarrow \mathcal{C}(A)$ of Theorem 2 .

In particular, this bundle admits no continuous cross-sections as soon as $A$ has no valuations, as in the case where $A=B(H)$ with $\operatorname{dim}(H)>2$.

The case where $A=B(H)$ with $\operatorname{dim}(H)>2$ is the content of the original KochenSpecker theorem [23].

PROOF. To prove this, we first give the external description of points of a pointfree space $\underline{Y}$ in a sheaf topos $\operatorname{Sh}(X)$. The subobject classifier in $\operatorname{Sh}(X)$ is the sheaf $\underline{\Omega}: U \mapsto \mathcal{O}(U)$, in terms of which a point of $\underline{Y}$ is a frame map $\mathcal{O}(\underline{Y}) \rightarrow \underline{\Omega}$. Externally, the point-free space defined by the frame $\underline{\Omega}$ is given by the identity map $\operatorname{id}_{X}: X \rightarrow X$, so that a point of $\underline{Y}$ externally corresponds to a continuous cross-section $\sigma: X \rightarrow Y$ of the bundle $\pi: Y \rightarrow X$ (that is, $\pi \circ \sigma=\mathrm{id}_{X}$ ). In principle, $\pi$ and $\sigma$ are by definition frame maps in the opposite direction, but in the case at hand, namely $X=\mathcal{C}(A)$ and $Y=\Sigma$, the map $\sigma: \mathcal{C}(A) \rightarrow \Sigma$ may be interpreted as a continuous cross-section of the projection (19) in the usual sense. Being a cross-section simply means that $\sigma(C) \in \Sigma(C)$. As to continuity, by definition of the Alexandrov topology, $\sigma$ is continuous if and only if the following condition is satisfied:

$$
\text { for all } \mathcal{U} \in \mathcal{O}(\Sigma) \text { and all } C \subseteq D \text {, if } \sigma(C) \in \mathcal{U} \text { then } \sigma(D) \in \mathcal{U}
$$

Hence, given the definition of $\mathcal{O}(\Sigma)$, the following condition is sufficient for continuity: if $C \subseteq D$, then $\sigma(D)_{\mid C}=\sigma(C)$. However, this condition is also necessary. To explain this, let $\rho_{D C}: \Sigma(D) \rightarrow \Sigma(C)$ again be the restriction map. This map is continuous and open. Suppose that $\rho_{D C}(\sigma(D)) \neq \sigma(C)$. Since $\Sigma(D)$ is Hausdorff, there is an open neighbourhood $\mathcal{U}_{D}$ of $\rho_{D C}^{-1}(\sigma(C))$ not containing $\sigma(D)$. Let $\mathcal{U}_{C}=$ $\rho_{D C}\left(\mathcal{U}_{D}\right)$ and take any $\mathcal{U} \in \mathcal{O}(\Sigma)$ such that $\mathcal{U} \cap \mathcal{O}(\Sigma(C))=\mathcal{U}_{C}$ and $\mathcal{U} \cap \mathcal{O}(\Sigma(D))=$ $\mathcal{U}_{D}$. This is possible since $\mathcal{U}_{C}$ and $\mathcal{U}_{D}$ satisfy both conditions in the definition of $\mathcal{O}(\Sigma)$. By construction, $\sigma(C) \in \mathcal{U}$ but $\sigma(D) \notin \mathcal{U}$, so that $\sigma$ is not continuous. Hence $\sigma$ is a continuous cross-section of $\pi$ if and only if

$$
\sigma(D)_{\mid C}=\sigma(C) \quad \forall C \subseteq D .
$$

Now define a map $\lambda: A_{\text {sa }} \rightarrow \mathbb{C}$ by $\lambda(a)=\sigma\left(C^{*}(a)\right)(a)$, where $C^{*}(a)$ is the commutative unital $C^{*}$-algebra generated by $a$. If $b^{*}=b$ and $[a, b]=0$, then $\lambda(a+b)=\lambda(a)+\lambda(b)$ by (26), applied to $C^{*}(a) \subset C^{*}(a, b)$ as well as to $C^{*}(b) \subset$ $C^{*}(a, b)$. Furthermore, since $\sigma(C) \in \Sigma(C)$, the map $\lambda$ is dispersion-free.

Conversely, a valuation $\lambda$ defines a cross-section $\sigma$ by complex linear extension of $\sigma(C)(a)=\lambda(a)$, where $a \in C_{\mathrm{sa}}$. By the criterion (26) this cross-section is evidently continuous, since the value $\lambda(a)$ is independent of the choice of $C$ containing $a$.

The contrast between the pointlessness of the internal spectrum $\underline{\Sigma}$ and the spatiality of the external spectrum $\Sigma$ is quite striking, but easily explained: a point of $\Sigma$ (in the usual sense, but also in the frame-theoretic sense in the case where $\Sigma$ is sober) necessarily lies in some $\Sigma(C) \subset \Sigma$, and hence is defined (and dispersion-free) only 
in the 'context' $C$. For example, for $A=M_{n}(\mathbb{C})$, a point $\lambda \in \Sigma(C)$ corresponds to a map

$$
\lambda^{*}: \mathcal{O}(\Sigma) \rightarrow\{0,1\}, S \mapsto \lambda(S(C)),
$$

where $\mathcal{O}(\Sigma)$ is realized as in (17). Thus $\lambda^{*}$ is only sensitive to the value of $S$ at $C$.

To close, we examine the possible soberness of $\Sigma$ [32, Theorem 8], [35, Theorem 2.25].

PROPOSITION 4. The space $\Sigma$ is sober if A satisfies the ascending chain condition: every chain $C_{1} \subseteq C_{2} \subseteq \cdots$ of elements $C_{i} \in \mathcal{C}(A)$ converges, in that $C_{n}=C_{m}$ for all $n>m$.

The proof is straightforward, relying on the identification of points of $\Sigma$ with irreducible closed subsets of $S$ and the ensuing condition that $\Sigma$ is sober if and only if every irreducible closed subset of $S$ is the closure of a unique point [28, Section IX.3].

For example, this proposition implies that $\Sigma$ is sober for $A=M_{n}(\mathbb{C})$, and, more generally, for all finite-dimensional $\mathrm{C}^{*}$-algebras.

\section{References}

[1] C. A. Akemann, 'A Gelfand representation theory for $C^{*}$-algebras', Pacific J. Math. 39 (1971), $1-11$.

[2] B. Banaschewski and C. J. Mulvey, 'The spectral theory of commutative $\mathrm{C}^{*}$-algebras: the constructive Gelfand-Mazur theorem', Quaest. Math. 23 (2000), 465-488.

[3] B. Banaschewski and C. J. Mulvey, 'The spectral theory of commutative $\mathrm{C}^{*}$-algebras: the constructive spectrum', Quaest. Math. 23 (2000), 425-464.

[4] B. Banaschewski and C. J. Mulvey, 'A globalisation of the Gelfand duality theorem', Ann. Pure Appl. Logic 137 (2006), 62-103.

[5] N. Bohr, 'Discussion with Einstein on epistemological problems in atomic physics', in: Albert Einstein: Philosopher-Scientist (ed. P. A. Schilpp) (Open Court, La Salle, IL, 1969), pp. 201-241.

[6] F. Borceux, Handbook of Categorical Algebra. 3. Categories of Sheaves (Cambridge University Press, Cambridge, 1994).

[7] J. Butterfield and C. J. Isham, 'A topos perspective on the Kochen-Specker theorem: I. Quantum states as generalized valuations', Internat. J. Theoret. Phys. 37 (1998), 2669-2733.

[8] M. Caspers, C. Heunen, N. P. Landsman and B. Spitters, 'Intuitionistic quantum logic of an $n$-level system', Found. Phys. 39 (2009), 731-759.

[9] R. Choukri, E. H. Illoussamen and V. Runde, 'Gelfand theory for non-commutative Banach algebras', Q. J. Math. 53 (2002), 161-172.

[10] A. Connes, Noncommutative Geometry (Academic Press, San Diego, CA, 1994).

[11] A. Connes and M. Marcolli, Noncommutative Geometry, Quantum Fields and Motives (American Mathematical Society, Providence, RI, 2008).

[12] T. Coquand, 'About Stone's notion of spectrum', J. Pure Appl. Algebra 197 (2005), 141-158.

[13] T. Coquand and B. Spitters, 'Constructive Gelfand duality for $C^{*}$-algebras', Math. Proc. Cambridge Philos. Soc. 147 (2009), 339-344.

[14] J. Dauns and K. H. Hofmann, Representation of Rings by Sections, Memoirs of the American Mathematical Society, 83 (American Mathematical Society, Providence, RI, 1968). 
[15] J. M. G. Fell and R. S. Doran, Representations of ${ }^{*}$-algebras, Locally Compact Groups, and Banach*-algebraic Bundles, Vol. 2 (Academic Press, Boston, MA, 1988).

[16] M. P. Fourman and D. S. Scott, 'Sheaves and logic', in: Applications of Sheaves (Proc. Res. Sympos. Appl. Sheaf Theory to Logic, Algebra and Anal., Univ. Durham, Durham, 1977), Lecture Notes in Mathematics, 753 (Springer, Berlin, 1979), pp. 302-401.

[17] R. Goldblatt, Topoi, The Categorical Analysis of Logic (North-Holland, Amsterdam, 1984).

[18] C. Heunen, N. P. Landsman and B. Spitters, 'A topos for algebraic quantum theory', Comm. Math. Phys. 291 (2009), 63-110.

[19] P. T. Johnstone, Stone Spaces (Cambridge University Press, Cambridge, 1982).

[20] P. T. Johnstone, Sketches of an Elephant: a Topos Theory Compendium, Vol. 1 (Clarendon Press, Oxford, 2002).

[21] P. T. Johnstone, Sketches of an Elephant: a Topos Theory Compendium, Vol. 2 (Clarendon Press, Oxford, 2002).

[22] A. Joyal and M. Tierney, An Extension of the Galois Theory of Grothendieck, Vol. 51, Memoirs of the American Mathematical Society (American Mathematical Society, Providence, RI, 1984).

[23] S. Kochen and E. P. Specker, 'The problem of hidden variables in quantum mechanics', J. Math. Mech. 17 (1967), 59-87.

[24] P. Kruszyński and S. L. Woronowicz, 'A noncommutative Gelfand-Naimark theorem', J. Operator Theory 8 (1982), 361-389.

[25] N. P. Landsman, 'Poisson spaces with a transition probability', Rev. Math. Phys. 9(1) (1997), 29-57.

[26] N. P. Landsman, Mathematical Topics between Classical and Quantum Mechanics (Springer, Berlin, 1998).

[27] N. P. Landsman, 'Between classical and quantum', in: Handbook of Philosophy of Science, Philosophy of Physics, 2 (eds. J. Earman and J. Butterfield) (Elsevier, Amsterdam, 2007), pp. 417-553.

[28] S. Mac Lane and I. Moerdijk, Sheaves in Geometry and Logic (Springer, Berlin, 1992).

[29] C. J. Mulvey, 'A noncommutative Gelfand-Naimark theorem', Math. Proc. Cambridge Philos. Soc. 88 (1980), 425-428.

[30] M. L. G. Redhead, Incompleteness, Nonlocality and Realism: a Prolegomenon to the Philosophy of Quantum Mechanics (Clarendon Press, Oxford, 1987).

[31] F. W. Shultz, 'Pure states as a dual object for $C^{*}$-algebras', Comm. Math. Phys. 82 (1981/82), 497-509.

[32] B. Spitters, 'The space of measurement outcomes as a spectrum for non-commutative algebras', EPTCS, doi: http://dx.doi.org/10.4204/EPTCS.26.12.

[33] B. van den Berg and C. Heunen, 'Noncommutativity as a colimit'. Appl. Categ. Structures, doi: 10.1007/S10485-011-9246-3.

[34] S. Vickers, Locales and Toposes as Spaces, Handbook of Spatial Logics, 8 (eds. M. Aiello, I. E. Pratt-Hartmann and J. F. A. K. van Benthem) (Springer, Berlin, 2007), pp. 429-496.

[35] S. Wolters, 'Contravariant vs covariant quantum logic: a comparison of two topos-theoretic approaches to quantum theory', arXiv:1010.2031.

\author{
CHRIS HEUNEN, Oxford University Computing Laboratory, \\ Wolfson Building, Parks Road, Oxford OX1 3QD, UK \\ e-mail: heunen@comlab.ox.ac.uk
}

NICOLAAS P. LANDSMAN,

Institute for Mathematics, Astrophysics, and Particle Physics, Radboud University Nijmegen, Heyendaalseweg 135,

6525 AJ Nijmegen, The Netherlands

e-mail: landsman@math.ru.nl 
BAS SPITTERS, Institute for Computer and Information Science,

Radboud University Nijmegen, Heyendaalseweg 135,

6525 AJ Nijmegen, The Netherlands

e-mail: spitters@cs.ru.nl

SANDER WOLTERS, Institute for Mathematics, Astrophysics, and Particle Physics, Radboud University Nijmegen, Heyendaalseweg 135, 6525 AJ Nijmegen, The Netherlands

e-mail: s.wolters@math.ru.nl 